\title{
Electromagnetic Compatibility Analysis of DVB-T and LTE Systems
}

\author{
Jurgis Aleksandravicius $^{1}$, Jevgenij Krivochiza ${ }^{1}$, Rimvydas Aleksiejunas ${ }^{1}$, Kestutis Svirskas ${ }^{1}$, \\ Mindaugas Zilinskas ${ }^{1,2}$ \\ ${ }^{I}$ Department of Radiophysics, Vilnius University, \\ Sauletekio al. 9, LT-10222 Vilnius, Lithuania \\ ${ }^{2}$ Communications Regulatory Authority of the Republic of Lithuania, \\ Algirdo st. 27A, LT-03219 Vilnius, Lithuania \\ jurgis.aleksandravicius@ff.vu.lt
}

\begin{abstract}
In 2012 the Communications Regulatory Authority of Lithuania reallocated 790-862 MHz band used by terrestrial TV operators for mobile communications purposes following an International Telecommunication Union decision. The aim of this paper is to experimentally investigate electromagnetic compatibility of Digital Video Broadcasting Terrestrial receivers operating below $790 \mathrm{MHz}$ band with LTE base stations operating in $800 \mathrm{MHz}$ band and derive an analytical model suitable for further electromagnetic compatibility analysis. An experimental testbed using LTE hardware and DVB-T receivers was used for evaluation of adjacent channel LTE downlink signal interference impact on digital TV system. Also, effect of using third party hardware such as band-pass filters and wideband preamplifiers on interference was analyzed. The results obtained provide protection ratio and overloading threshold for the digital TV receivers in different operating scenarios which serve as a reference guide for regulatory authority, mobile operators and terrestrial TV providers.
\end{abstract}

Index Terms-Electromagnetic compatibility; LTE; UHF communication; radio frequency interference.

\section{INTRODUCTION}

Following International Telecommunication Union decision to repurpose Digital Dividend 1 (791-862 MHz) for mobile telecommunications in Europe the Communications Regulatory Authority of the Republic of Lithuania divided the band into six frequency duplex blocks with downlink and uplink bands. Each mobile operator acquired two duplex blocks, allowing their LTE systems to operate in total $2 \times 5 \mathrm{MHz}$ frequency band for downlink and $2 \times 5 \mathrm{MHz}$ frequency band for uplink communications. The UHF band of the Digital Terrestrial Television (DTT) system was reduced by 9 channels due to increased spectral efficiency after analog-to-digital switchover.

Electromagnetic compatibility (EMC) issues of two systems operating in adjacent frequency bands emerged as DVB-T (Digital Video Broadcasting - Terrestrial) receivers currently in use are still sensitive for the channels, which are

Manuscript received 21 December, 2015; accepted 28 March, 2016.

This research was funded and performed in cooperation with the Communications Regulatory Authority of the Republic of Lithuania for radio communications expansion in the $800 \mathrm{MHz}$ frequency band. reallocated for already commercially operating LTE mobile system. European regulators have been actively investigating coexistence problems of DTT and LTE systems [1]-[5]. Most efforts were put into investigating degradation of DTT service quality, putting technical requirements for LTE operators.

This paper firstly aims to experimentally and analytically investigate adjacent channel interference impact on modern DVB-T receivers with MPEG4 transport stream support. Secondly, to investigate impact of additional hardware, e.g. signal preamplifiers and filters available in commercial market on reception quality and give recommendations for DTT operators on effective ways to mitigate adjacent interference on end-user side. The experiment includes measurements of protection ratio with different DVB-T signal modulation schemes, code rates, guard intervals, frequency channels and received power combined with variations of LTE signal bandwidth and operating frequency. The experimental results are compared against analytical model, which can be used in further evaluations of EMC in different spectrum allocation scenarios [6].

The paper is organized as follows. Section II presents technical parameters of the systems and testbed overview. Section III is devoted to methods and criteria for evaluation of interference impact. The analytical model is described in Section IV. Experimental results and analysis follows in Section V.

\section{SYSTEM PARAMETERS}

The investigation aimed to evaluate performance of three different commercially available DVB-T receivers in the presence from strong adjacent-channel interference of downlink LTE Frequency division duplex signal. The measurements were conducted on a wired testbed in Telecommunication Research Center at Vilnius University which has been set up according to the Electronic Communications Committee (ECC) Report [1]. Radio frequency (RF) inputs of DVB-T receivers were fed over wired environment through power combiner with signals from DVB-T and LTE signal generators. Three types of band-pass filters (denoted FILT1, FILT2, FILT3) and wideband signal preamplifiers (denoted AMP1, AMP2, 
AMP3) were considered in this measurement campaign. The filters are designed to suppress LTE interference and have passband from $5 \mathrm{MHz}$ to $790 \mathrm{MHz}$ with varying quality of signal suppression above this band. Conversely, all three preamplifiers are sensitive to signals up to $862 \mathrm{MHz}$ frequency. Their gains are $14 \mathrm{~dB}, 18 \mathrm{~dB}$ and $25 \mathrm{~dB}$, respectively. Interference and signal power have been measured at RF inputs of the filters and amplifiers. A more detailed block scheme of the testbed is depicted in Fig. 1.

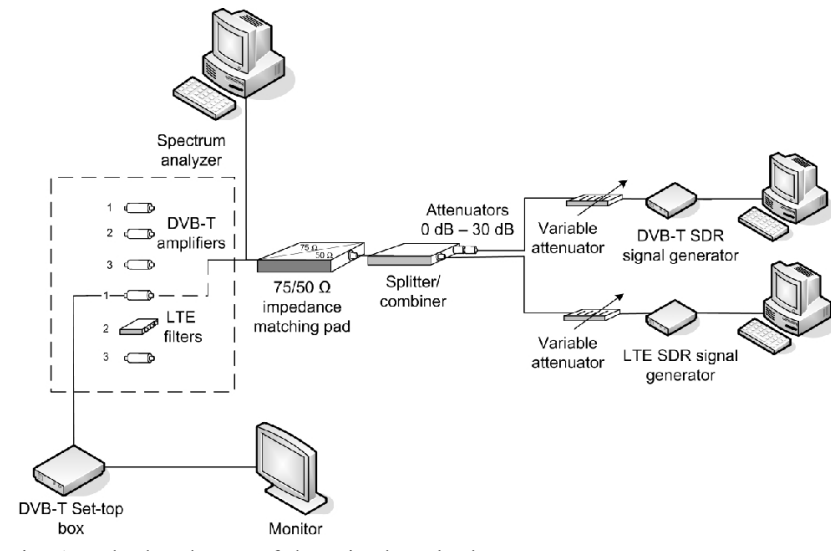

Fig. 1. Block scheme of the wired testbed.

Pre-recorded DVB-T and LTE Base Station (BS) baseband signals were transmitted by software defined radios (SDR). Examples of generated passband signal spectra are shown in Fig. 2 and Fig. 3. Both $10 \mathrm{MHz}$ and $5 \mathrm{MHz}$ bandwidth LTE BS signals comply with European Conference of Postal and Telecommunications Administrations (CEPT) spectrum mask [1].

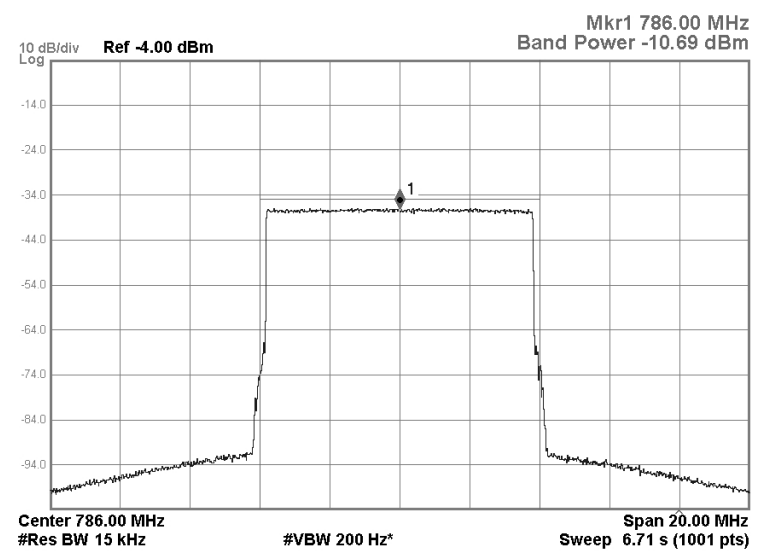

Fig. 2. DVB-T RF signal spectrum mask.

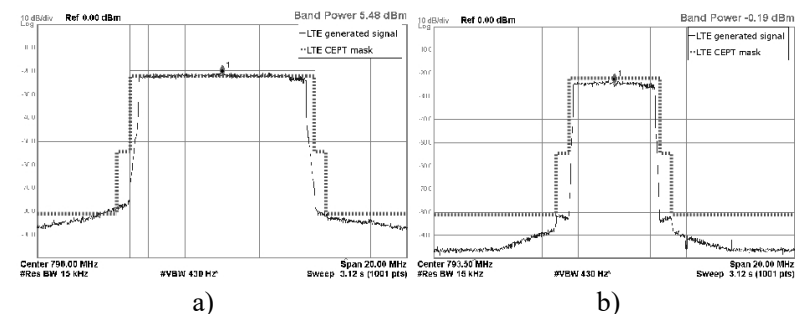

Fig. 3. LTE $10 \mathrm{MHz}$ (a) and $5 \mathrm{MHz}$ (b) fully loaded BS RF signal spectrum and CEPT spectrum mask.

Performance of DVB-T receivers was evaluated in a number of system parameter combinations, summarized in Table I. DVB-T signal containing MPEG-4 transport stream was fully occupied by payload bits on each modulation scheme. The generated LTE BS signal occupies all available resource blocks on $5 \mathrm{MHz}$ and $10 \mathrm{MHz}$ frequency bands.

TABLE I. DVB-T AND LTE SIGNAL MEASUREMENT PARAMETERS.
\begin{tabular}{|c|c|}
\hline Parameter & Value \\
\hline $\begin{array}{c}\text { DVB-T signal center } \\
\text { frequencies (channel } \\
\text { number) }\end{array}$ & $714 \mathrm{MHz}-786 \mathrm{MHz}(51-60)$ \\
\hline $\begin{array}{c}\text { DVB-T signal } \\
\text { bandwidth }\end{array}$ & $8 \mathrm{MHz}$ \\
\hline $\begin{array}{c}\text { DVB-T signal } \\
\text { modulation schemes }\end{array}$ & 64 QAM, $16 \mathrm{QAM}$, QPSK \\
\hline $\begin{array}{c}\text { DVB-T signal coding } \\
\text { rates }\end{array}$ & $-70 \mathrm{dBm},-60 \mathrm{dBm},-50 \mathrm{dBm}$, \\
\hline $\begin{array}{c}\text { DVB-T signal power } \\
(P \text { DVBT })\end{array}$ & $-40 \mathrm{dBm},-30 \mathrm{dBm},-20 \mathrm{dBm}$ \\
\hline $\begin{array}{c}\text { LTE signal bandwidth } \\
\text { LTE signal center } \\
\text { frequencies for } 5 \mathrm{MHz} \\
\text { band }\end{array}$ & $\begin{array}{c}793.5 \mathrm{MHz}, 798.5 \mathrm{MHz}, 803.5 \mathrm{MHz}, \\
808.5 \mathrm{MHz}, 813.5 \mathrm{MHz}, 818.5 \mathrm{MHz}\end{array}$ \\
\hline $\begin{array}{c}\text { LTE signal center } \\
\text { frequencies for } 10 \mathrm{MHz} \\
\text { band }\end{array}$ & $796 \mathrm{MHz}, 806 \mathrm{MHz}, 816 \mathrm{MHz}$ \\
\hline
\end{tabular}

Three DVB-T receivers (Set-top boxes) have been evaluated in this campaign, further referred to as STB1, STB2 and STB3. Receiver STB1 was a heterodyne receiver with discrete electronic components. The other two receivers were "silicon-based", i.e. using a custom electronic chip for TV signal demodulation.

\section{Methods of Performance Evaluation}

There are a number of criteria for evaluating interference impact. Ideally performance of DVB-T receivers should be evaluated by observing bit error rate (BER) after convolutional decoder or the number of transport stream errors. However, it is usually not possible to obtain such measurements in commercial DVB-T receivers because of the absence of relevant output ports. The preferred way is instead to evaluate performance by picture failure (PF), which is defined as the number of visually observed picture artefacts during a certain period [1], [2]. The use of forward error correction (FEC) coding in digital systems causes a sudden decline of picture quality when interference power increases. The measurements in this campaign were determined by ensuring zero PF events over a period of 30 seconds.

Measurements were taken with fixed DVB-T signal power values summarized in Table I. On each measurement the maximum non-interfering LTE signal power $P_{L T E_{\max }}$ was determined and signal-to-interference ratio (SIR) was evaluated. SIR here corresponds to the ratio (expressed in dB) of DVB-T and LTE signal powers denoted here as $P_{D V B T}$ and $P_{L T E_{\max }}$, respectively. These values were measured at receiver's RF input. When an additional filter or an amplifier was connected to receiver's RF input, signal power values were measured at the input of that additional component. Performance of DVB-T receivers was quantified using two indicators, protection ratio (PR) and overloading threshold (OT).

Overloading threshold is defined as the power of adjacent channel interfering signal above which the receiver begins to respond non-linearly. Depending on the receiver it may or 
may not fail once the interfering signal is greater than overloading threshold. The overloading threshold can be derived from variation of $P_{L T E_{\max }}$ against $P_{D V B T}$. Overloading is a property of the receiver.

The procedure of evaluating the Overloading threshold is defined as follows. Increasing values of $P_{\mathrm{DVBT}}$ are set and corresponding $P_{L T E_{\max }}$ values are measured. At some point $P_{L T E_{\max }}$ values stop increasing, i.e. an interfering signal of certain power overloads the receiver. Beyond that point reception of DVB-T transmission will not be decoded irrespective of the magnitude of $P_{\mathrm{DVBT}}$.

Protection ratio is the minimum value of SIR required in order to achieve specified reception quality under specified conditions. If $P_{\mathrm{DVBT}}$ and $P_{L T E_{\max }}$ are expressed in $\mathrm{dBm}$ then

$$
P R=S I R_{\min }=P_{\mathrm{DVBT}}-P_{\mathrm{LTE}_{\max }}(\mathrm{dB}) .
$$

PR and OT have been evaluated in various conditions corresponding to signal parameters that are summarized in Table I.

\section{Analytical Model of PERFormance EVAluation}

The analytical model is based on frequency-dependent rejection factor (FDR) calculations [7]. In this work properly defined DVB-T and LTE signal spectrum masks (Fig. 4) are used to match the experimental data. The discrete formula for calculating FDR is

$$
\begin{gathered}
F D R\left(f_{\text {off }}\right)=10 \log _{10} \times \\
\sum_{f=0}^{f_{\max }}\left[P(f)_{\mathrm{DVBT}} \times P\left(f+f_{\text {off }}\right)_{\mathrm{LTE}}\right] \\
\sum_{f=0}^{f_{\max }} P(f)_{\mathrm{DVBT}}
\end{gathered}
$$

where $P(f)_{\mathrm{DVBT}}$ is DVB-T signal spectrum mask, $P(f)_{\mathrm{LTE}}$ LTE signal spectrum mask, $f_{\max }$ is maximum integration frequency offset of DVB-T spectrum mask and $f_{\text {off }}$ is frequency offset between center frequency of victim and interfering signals.

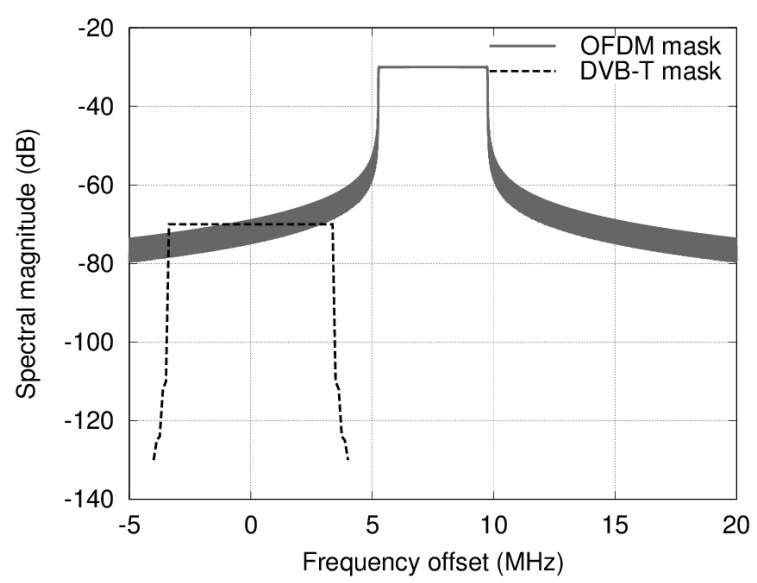

Fig. 4. Simulation OFDM signal spectrum masks for LTE and DVB-T signals. $P_{\mathrm{DVBT}}=-70 \mathrm{dBm}, P_{\mathrm{LTE}}=-30 \mathrm{dBm}$.

In Fig. 4 two simulated OFDM spectrum masks are shown. Since information about RF selectivity of DVB-T receivers is unknown, the DVB-T spectrum mask used for FDR calculations is based on the shape of generated DVB-T signal spectrum.

\section{RESULTS}

\section{A. Overloading Threshold}

Figure 5 shows variation of $P_{L T E_{\max }}$ against wanted signal power in receiver STB1. The three highest frequency TV channels are compared.

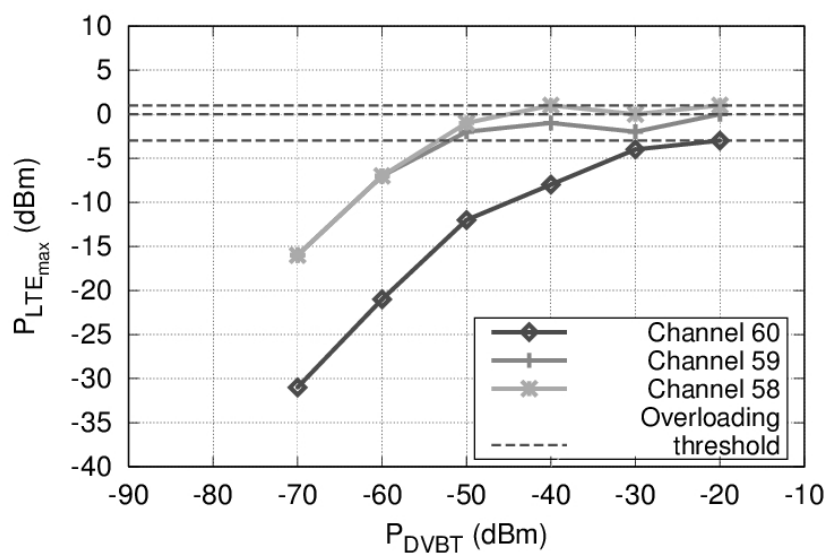

Fig. 5. Overloading threshold vs. DVB-T signal power in receiver STB1. LTE parameters: 64 QAM, center frequency 793.5 MHz.

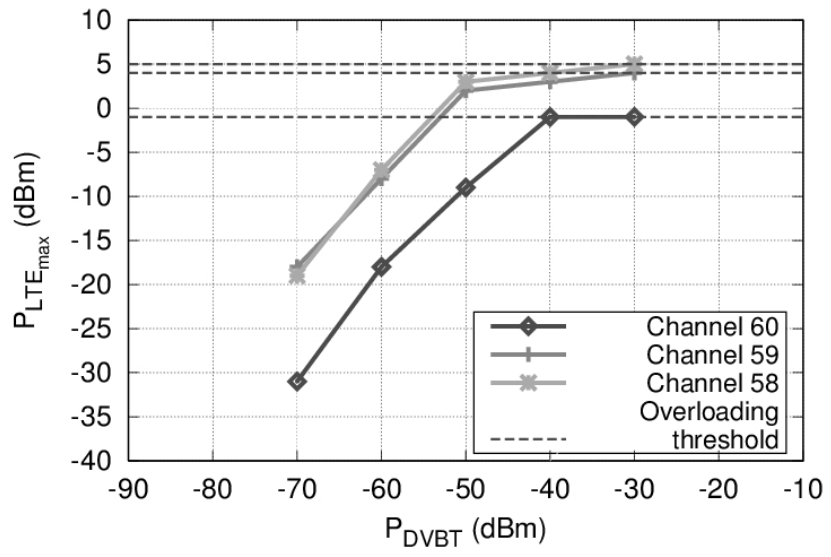

Fig. 6. Overloading threshold vs. DVB-T signal power in receiver STB2. LTE parameters: 64 QAM, center frequency 793.5 MHz.

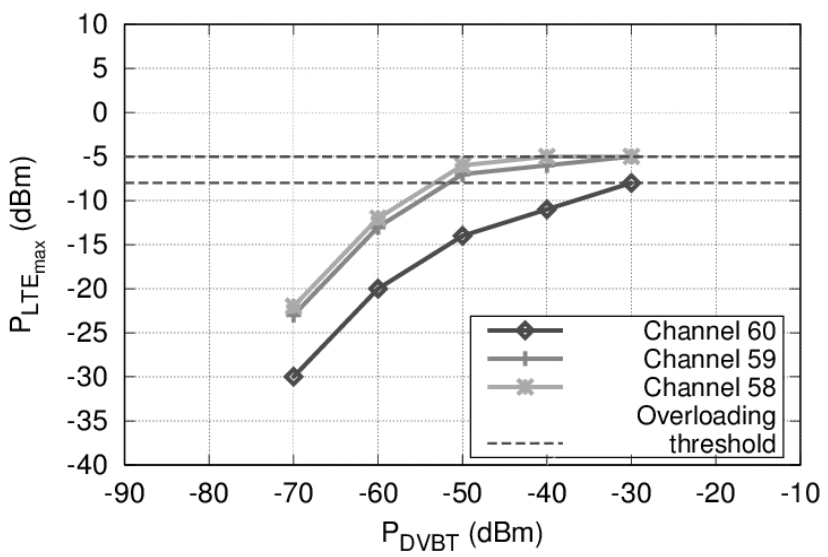

Fig. 7. Overloading threshold vs. DVB-T signal power in receiver STB3. LTE parameters: 64 QAM, center frequency $793.5 \mathrm{MHz}$.

It demonstrates the non-linear behavior of receiver frontend when a strong out-of-band interfering signal is present. 
Ideally $P_{L T E_{\max }}$ should be linearly dependent on wanted signal power $P_{D V B T}$. However it reaches a threshold where an increase in wanted signal power will not result in improved reception conditions if the threshold is exceeded. The threshold generally depends on wanted signal frequency and parameters of interfering signal.

Figure 6 and Fig. 7 show results of measurements on receivers STB2 and STB3 in the same conditions. It can be seen that STB2 offers higher overloading threshold against adjacent channel interference.

\section{B. Protection Ratio}

The measurement campaign resulted in an extensive amount of data recorded in various conditions. It was perhaps of the most interest to compare performances of receivers with and without an additional filter or an amplifier. In these results $\mathrm{PR}$ is negative since adjacent channel interference power can be greater than the wanted signal power. A lower PR value indicates a lower requirement for protection against unwanted interference from LTE base stations and consequently better reception conditions.

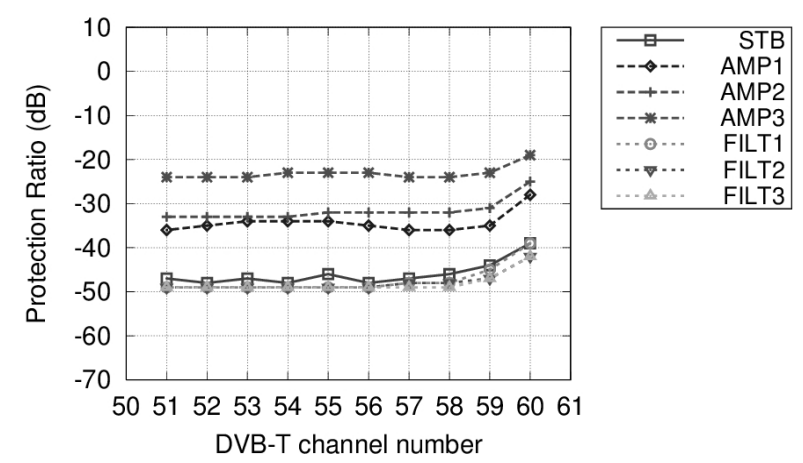

Fig. 8. Protection ratio vs. DVB-T frequency channel number. LTE parameters: 64 QAM, center frequency 793.5 MHz. PDVBT $=-60 \mathrm{dBm}$.

Figure 8 shows variation of PR against DVB-T carrier frequency expressed as the channel number. Firstly, it is evident that required protection is always larger when an amplifier is connected to the RF input of the receiver (data lines AMP1, AMP2, AMP3) as compared to results with no amplifier (data line STB). On the contrary, required protection can be slightly reduced by connecting a LTE filter to the receiver (data lines FILT1, FILT2, FILT3).

Secondly, these results reveal the dependence of PR on frequency offset. When $f_{\mathrm{LTE}}=793.5 \mathrm{MHz}$ and DVB-T is transmitted on the channels 59 and 60 (channel edge separation $9 \mathrm{MHz}$ and $1 \mathrm{MHz}$ respectively) the observed increase in PR is up to $8 \mathrm{~dB}$. In TV channels further away from LTE band PR does not depend on channel edge separation.

\section{Comparison of FDR and PR}

Figure 9 shows the comparison of measured PR data with analytically calculated FDR values. Analytical FDR curves closely follow experimental data. Both calculated FDR and measured PR values decrease when frequency offset increases as less interfering signal power falls within receiver passband. Measured PR values decrease accordingly to (1) as LTE power required for PF event to occur $\left(P_{L T E_{\max }}\right)$ increases with increasing frequency offset We suggest that FDR can be used as an indicator for characterization of PR dependence on frequency offset when estimating potential interference in new frequency bands in case experimental measurements are not available [6].

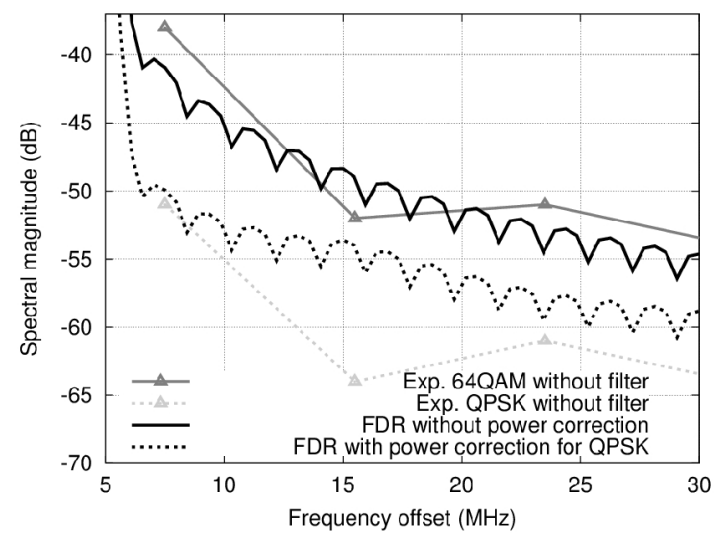

Fig. 9. Measured PR and analytically calculated FDR dependencies on center frequency offset between victim and interfering signals.

\section{CONCLUSIONS}

We have presented results from a measurement campaign of susceptibility of DVB-T receivers to interference from LTE BS signals and suggested a method for estimation of DVB-T receiver protection ratio dependence on frequency offset between DVB-T and interfering LTE signals.

Among the main findings is that a LTE BS operating in frequencies closest to DVB-T band can cause strong DTT service quality degradation on channels 58, 59 and 60 . Furthermore, if a preamplifier is used LTE interference impact increases dramatically. An analytical method based on FDR calculation that allows predicting PR variation with frequency offset is presented and compared with measurements. The results will be particularly valuable to radio regulatory authorities, mobile operators and DTT operators for planning new network installations or solving particular interference issues in operational networks.

\section{REFERENCES}

[1] ECC Report 148: DVB-T performance in the presence of LTE, Electronic Communications Committee within the European Conference of Postal and Telecommunications Administrations, France, June 2010.

[2] OFCOM Technical Report: Technical analysis of interference from mobile network base stations in the $800 \mathrm{MHz}$ band to digital terrestrial television, ERA Technology Limited, UK, June 2011.

[3] G. Baruffa, M. Femminella, F. Mariani, G. Reali, "Protection ratio and antenna separation for DVB-T/LTE coexistence issues", IEEE Communications Letters, vol. 17, no. 8, pp. 1588-1591, 2013. [Online]. Available: http://dx.doi.org/10.1109/LCOMM.2013. 070113.130887

[4] C. Elofsson, C. Karlsson, C. Beckman, "On the interference between digital terrestrial television and mobile broadband in the Swedish 800 $\mathrm{MHz}$ band", Int. Symposium on Electromagnetic Compatibility (EMC Europe 2014), 2014. [Online]. Available: http://dx.doi.org/10.1109/EMCEurope.2014.6930963

[5] K. Sakic, M. Gosta, "Measurements on the influence of the LTE system on DVB-T reception", in Proc. IEEE ELMAR 2012, pp. 247250 .

[6] ITU: Digital Dividend Insights for Spectrum Decisions, ITU, Switzerland Geneva, 2012.

[7] W. Kuebler, S. Cameron, "The definition of Frequency-Dependent rejection", IEEE Trans. Electromagnetic Compatibility, no. 4, pp. 349-350, 1979. 